\title{
Arthritis Identification from Multiple Regions by X-Ray Image Processing
}

\author{
Hunza Hayat ${ }^{1}$, Syed Omer Gilani ${ }^{1}$ and Mohsin Jamil ${ }^{2}$ \\ ${ }^{1}$ Department of Biomedical Engineering and Sciences \\ ${ }^{2}$ Department of Robotics and Artificial Intelligence \\ School of Mechanical and Manufacture Engineering (SMME), \\ National University of Sciences and Technology (NUST), \\ Sector H-12, Main Campus, Islamabad Pakistan \\ hunza.hayat@gmail.com,omer@smme.nust.edu.pk,mohsin@smme.nust.edu.pk
}

\begin{abstract}
The $x$-ray image processing is an effective technique to distinguish between the major types of arthritis: Osteoarthritis Arthritis (OA) and rheumatoid Arthritis (RA). The degenerative bone disorders are diagnosed using X-ray detection. X-ray scans alone are insufficient to detect the type of arthritis. Image processing can aid in improving the diagnosis. The classification is done on the basis of differentiation in the region properties and boundary areas that can be identified using MATLAB. These properties were used to better understanding the variation in the knee, hands and neck region. The study holds potential as a diagnostic tool for arthritis identification through $x$-rays. It could pave way to differential diagnosis in future.
\end{abstract}

Keywords: Rheumatoid arthritis (RA), Osteoarthritis( $0 A)$, image processing, region property, X-ray image processing, major axis length, minor axis length, normal hand image, comparative image plot, gray scale, Gaussian filtering, X-ray image, neck region ,normalized second central moment, normal hand

\section{Introduction}

Arthritis is bone joint inflammation disease which has multiple types and conditions. The two most recurrent types are Osteoarthritis (OA) and Rheumatoid Arthritis (RA). Osteoarthritis $(\mathrm{OA})$ is a heritable as well as non-heritable, degenerative disabling condition while Rheumatoid Arthritis (RA) is heritable, auto-immune disease. Multiple factors are involved in the onset and gradual loss of the articular cartilage in these two major forms of arthritis. The identification of these diseases is done by X-Ray but the conventional method lacks in precision and accurate analysis thus image processing can be conducted to assist this issue.

According to recent studies, a series of inflammatory processes may lead to initiation and propagation of OA (Marla J. Steinbeck, 2007). According to World Health Organization (WHO) osteoarthritis is a serious disabling disease, statistically 1 in 10 developed countries are under its influence. Worldwide estimates of $9.6 \%$ men and $18.0 \%$ women over 60 years of age have symptomatic OA. Osteoarthritis is a degenerative joint disease and the most common type of arthritis as well. 0.5 to $1 \%$ of adults are subject to RA with between 5 and 50 per 100,000 people prone to this condition every year. (Symmons, D., 2002)

Osteoarthritis majorly affects cartilage covering the bone ends causing bones underneath to rub together, resulting in pain, swelling, and loss of motion of the joints. With time, joints lose their normal shape and osteophytes (bone spurs) grow on the edges.

Received (June 5, 2017), Review Result (September 30, 2017), Accepted (October 5, 2017) 
Broken bits of bone or cartilage float inside the joint spaces. In RA synovitis causes tissue tethering leading to movement inability and erosion surface erosion resulting in bone deformities (Majithia, V., and Geraci, S. A. 2007).

The only means of detection of arthritis is by X-Ray studies in Pakistan thus there is room for research on better or improved methods of assessment and prediction of the disease type and degree. There is often a mismatch between the stage of OA depicted and the actual degree of pain and disability observed by the patient while the X-Ray cannot solely depict clear distinction amongst different forms of arthritis.

MRI (magnetic resonance imaging) on the other hand provides high-resolution internal body tissues images. A strong magnet passes a force through the body to create an image. MRI tests are used if x-ray analysis is insufficient and other joint tissues such as a ligament are damaged. NIAMS-researchers are working on a technique called micro computed tomography (micro CT). It is a high-resolution, three-dimensional x-ray image creating technique which can identify the content and distribution of proteoglycans. This is involved in studying the thickness and composition of cartilage, thus helps to monitor the progress and treatment of OA. The technique is limited to animal studies and success rate in humans is still a far cry. The diagnosis by conventional X-Ray is a less expensive technique as compared to MRI and micro $\mathrm{CT}$, thus by designing a detection algorithm on $\mathrm{X}$-Ray images in MATLAB the identification can be made more accurate.

\section{Material and Method}

\section{Patient Characterization}

The study is conducted on the available x-ray image dataset at rheumatoid.images.org with a total of $>60$ images of knee, hand and neck of RA, OA and normal individuals.

\section{Image Characterization}

The salient feature of $\mathrm{OA}$ is the absence of synovial fluid from the joints while it is thickened and forms nodular structure in the case of RA; well evident through knee $\mathrm{x}$ rays. The hand bones (phalanges) become disoriented leaning outwards or curling up on one another in case of RA while the swollen joints with bone spurring on edges. The spinal disks have reduced spaces in OA while the cervical spine (neck) region is more likely influenced by RA than lower back.

\section{Image Processing Application}

The digital image processing is done by acquiring the dataset, storing and compressing them to initiate the processing techniques. The features are slightly or extremely evident from the x-ray depending upon the stage and type of arthritis. The process is as follows;

- Image Acquisition: image is acquired by sampling

- Gray scale conversion/Thresholding: the image is converted to gray scale if needed/ the image is made binary using the thresholding function

- Compression: image is resized and its archive size is reduced

- Median Filter/Gaussian Filtering: the image is made smooth

- Region properties: The region properties such as Centroid, extrema, orientation, major axis length, minor axis length and eccentricity are applied to the images in order to obtain statistical data

- Feature Extraction: Joint location, orientation, circular features and space detection is done

- Image Recognition: classification amongst OA, RA and normal. 


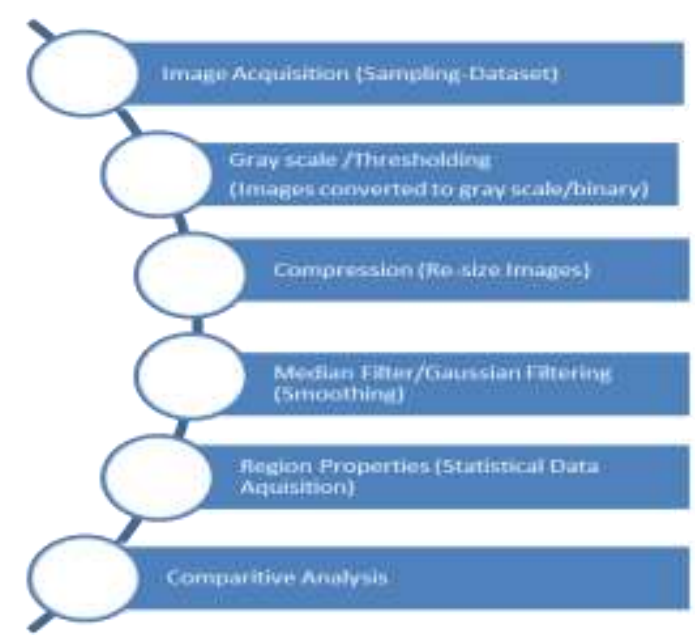

Figure 1. Data Analysis Flow Diagram

\section{Median Filtering/Gaussian Filtering}

The digital non-linear filtering to remove the unwanted noise is done by median filtering. It is a part of the image preprocessing protocol and is often used in the case of $\mathrm{x}$ ray image processing for edge detection. The Gaussian filtering involves convolution which is like multiplication of two input images to produce modified output or crosscorrelation between inputs. The self-Gaussian filter helps identify edges and make the image smooth.

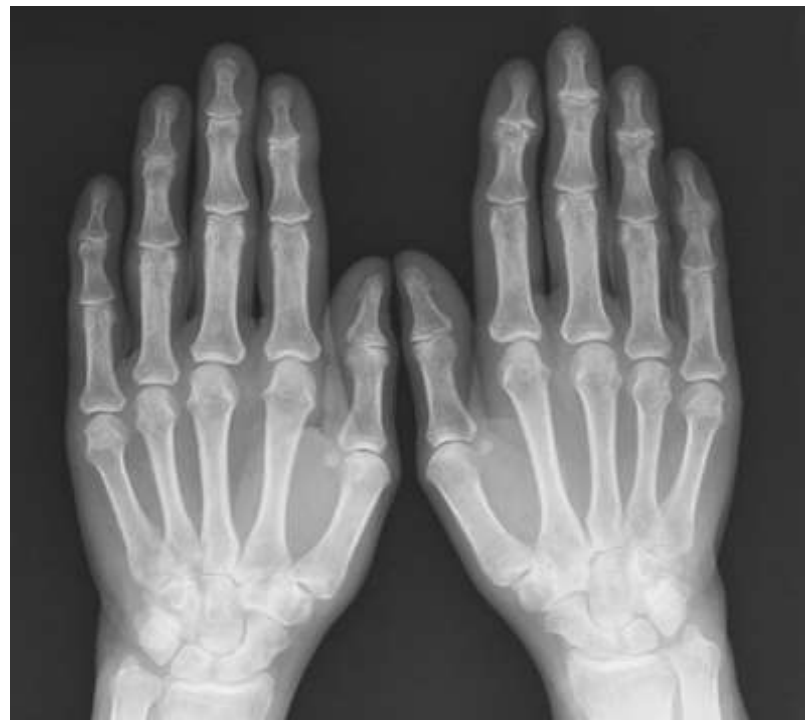

Figure 2. Normal Hands Image 


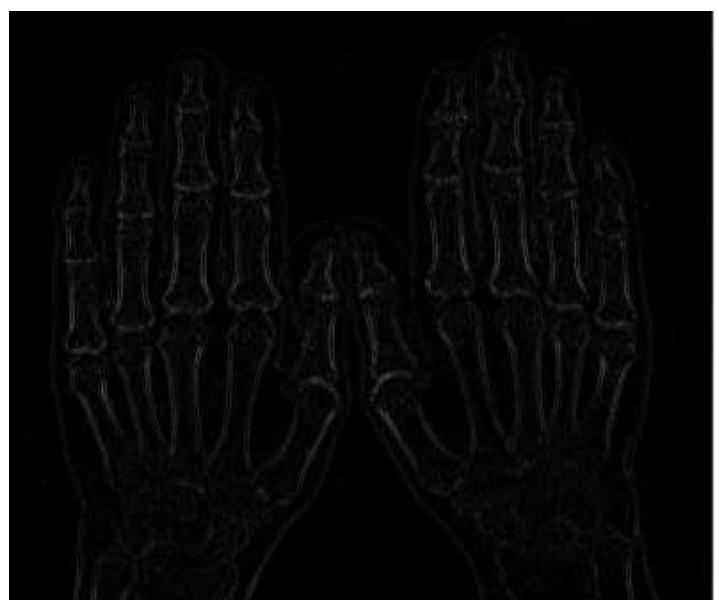

Figure 3. Resized, Gray Scaled and Gaussian Filtered Normal Hands Image

\section{Region Properties}

The specific properties related to connected regions are brought under focus using the region prop function in Matlab.

- Centroids- The center of mass of connected region is identified using the centroid command. The $x-y$ coordinates of the central point are marked by means of blue dots on the image.

- Orientation- The angle between the $\mathrm{x}$ - axis and major axis ranging from -90 to 90 degrees.

- Major Axis Length- The length (in pixels) of the major axis with same normalized second central moments as the region.

- Minor Axis Length - The length (in pixels) of the minor axis with same normalized second central moments as the region.

- Extrema- 8-by-2 matrix that specifies the extreme points in the region. The top left and top right for example will be identical.

- Eccentricity-Distinguishes between circle and line segments

The following is an example of the region properties command applied to the normal hands image to conduct statistical analysis.

The image is converted to binary state to apply the region properties.

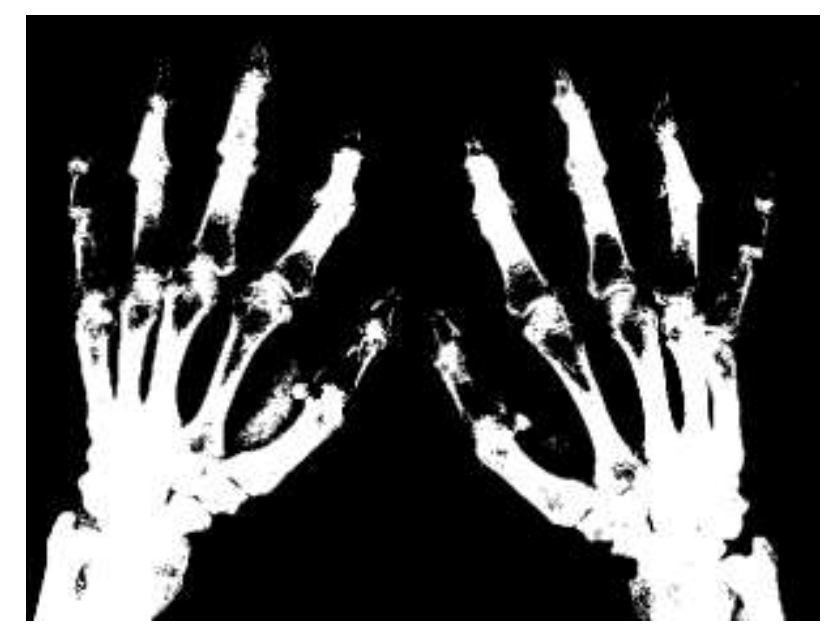

Figure 4. Normal Hands Binary Image 


\begin{tabular}{|c|c|c|c|c|c|c|}
\hline \multicolumn{2}{|c|}{ Centroid } & \multirow{2}{*}{$\frac{\text { NajorAxisLength }}{612.38}$} & \multirow{2}{*}{$\frac{\text { MinorAxisLength }}{268.53}$} & \multirow{2}{*}{$\begin{array}{l}\text { Bocentricity } \\
0.89873\end{array}$} & \multirow{2}{*}{$\frac{\text { Orientation }}{72.926}$} & \multirow{2}{*}{$\begin{array}{l}\text { Extrema } \\
\text { [8x2 double }\end{array}$} \\
\hline 180.38 & 501.13 & & & & & \\
\hline 74.966 & 189.01 & 23.939 & 16.236 & 0.73487 & -6.4935 & [ $8 \times 2$ double] \\
\hline 75.864 & 264.89 & 110.64 & 20.646 & 0.98244 & -88.259 & [8x2 double] \\
\hline 69.5 & 237.5 & 3.0551 & 1.1547 & 0.92582 & 45 & [8x2 double] \\
\hline 70 & 199.5 & 2.3094 & 1.1547 & 0.86603 & 90 & [ $8 \times 2$ double] \\
\hline 71 & 178.5 & 2.3094 & 1.1547 & 0.86603 & 90 & [8x2 double] \\
\hline 71 & 342 & 3.4641 & 1.1547 & 0.94281 & 90 & [8x2 double] \\
\hline 71.5 & 601.5 & 3.0551 & 1.1547 & 0.92582 & -45 & [8x2 double] \\
\hline 73 & 137 & 1.1547 & 1.1547 & 0 & 0 & [ $8 \times 2$ double] \\
\hline 73 & 179 & 3.4641 & 1.1547 & 0.94281 & 90 & [ $8 \times 2$ double] \\
\hline 73 & 231 & 1.1547 & 1.1547 & 0 & 0 & [ $8 \times 2$ double] \\
\hline 73.4 & 330.6 & 4.3631 & 2.0272 & 0.8855 & 74.518 & [8x2 double] \\
\hline 74 & 606 & 1.1547 & 1.1547 & 0 & 0 & [ $8 \times 2$ double] \\
\hline 75 & 615 & 1.1547 & 1.1547 & 0 & 0 & [ $8 \times 2$ double] \\
\hline 76 & 179 & 1.1547 & 1.1547 & 0 & 0 & [8x2 double] \\
\hline 76 & 600 & 1.1547 & 1.1547 & 0 & 0 & [8x2 double] \\
\hline 79.393 & 610.21 & 12.091 & 6.6853 & 0.83324 & -71.016 & [ $8 \times 2$ double] \\
\hline 76 & 611 & 1.1547 & 1.1547 & 0 & 0 & [8x2 double] \\
\hline 77 & 203 & 1.1547 & 1.1547 & 0 & 0 & [ $8 \times 2$ double] \\
\hline 77.5 & 604 & 2.3094 & 1.1547 & 0.86603 & 0 & [ $8 \times 2$ double] \\
\hline
\end{tabular}

Figure 5. Statistical Data using Region Properties

\section{Boundary Area}

The boundary area command helps to create boundaries of the multiple segments in the image with the area mentioned that is closed within. The areas are encircled based on the region of connectivity. Furthermore the areas can be distinguished on the base of color and size. This improves the image analysis variables provided for image processing.

Following is the code for the centroid identification followed by area identification on the basis of regions of high connectivity. Then finally there is boundary identification alongside area with numerical value.

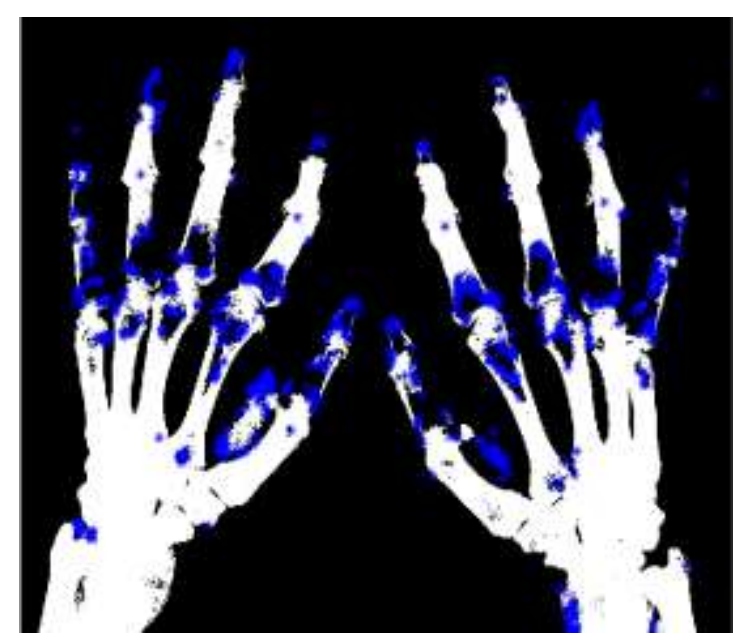

Figure 6. Centroid Identification on Normal Hands 


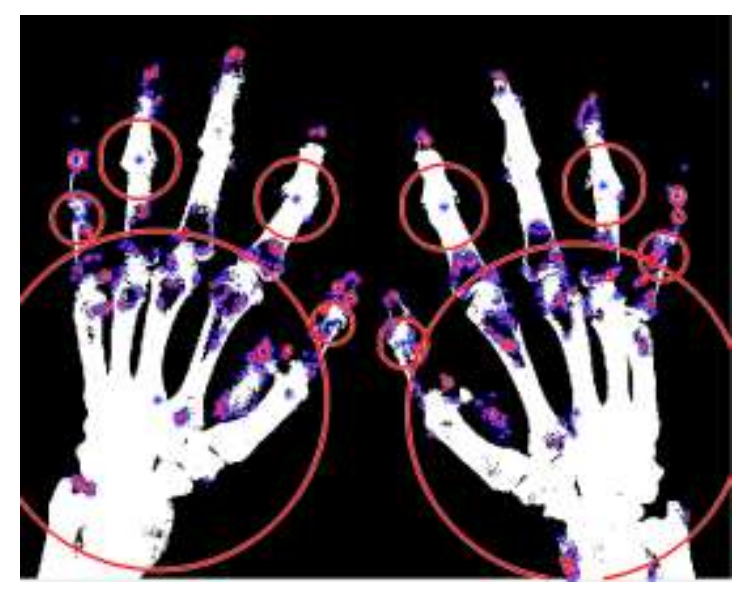

Figure 7. High Area Connectivity In Normal Hands

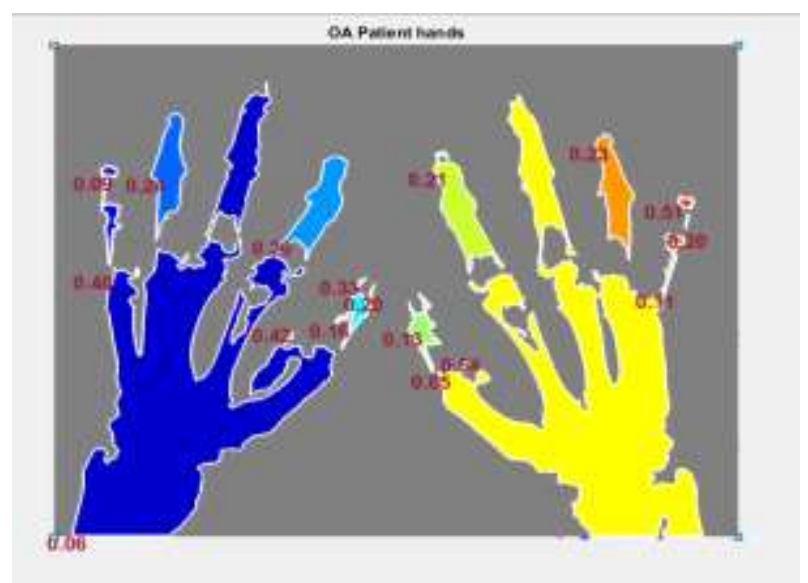

Figure 8. Boundary (area) Identification of Segmented Regions of Normal Hands

\section{Identification in Different Body Regions}

The above mentioned code was applied to the x-ray images of normal, OA and RA patient; knee, hand and neck regions. The results were plotted together for comparative analysis.

\section{Results}

The following figure shows the comparison between the knee images of normal, OA and RA patients. The connected region is larger in the normal as compared to the OA or RA as the bone disruption causes the bone spurring in both the diseases. The Centroids identified are more in case of OA as bone splinters are suspended around the region. The orientation is more evident in the case of RA and larger variations in area are projected by results 

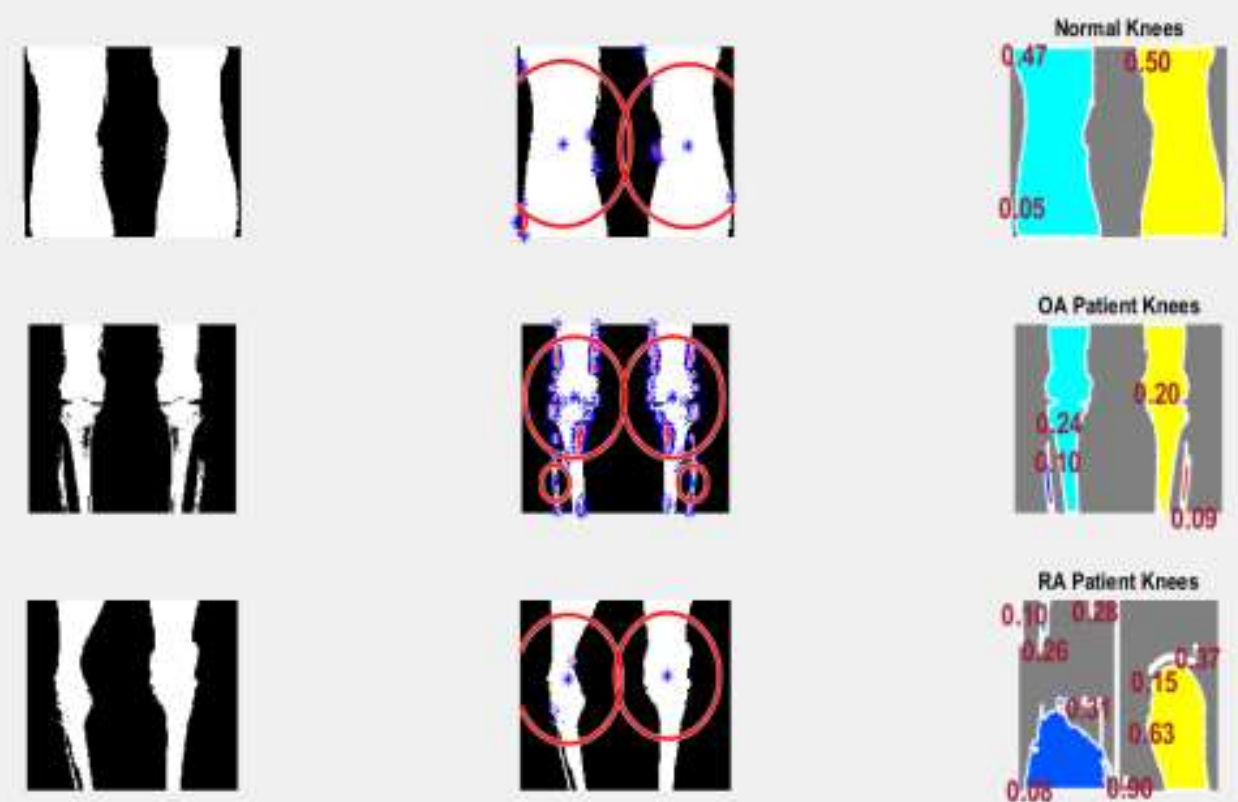

Figure 9. Comparative Image Plot for Normal, OA and RA Patient Knees

The hands of RA patients are one of the initial identification signs of the condition while they can be confused with those of OA patients as the orientation is disturbed in both conditions but the pattern is different. The symmetrical disorientation is in RA while there is no visible correlation of specified orientation in the case of OA. The number of centroids is more in RA as compared to OA. The increased change in orientation and structure can be used to identify the different conditions by this imaging technique..
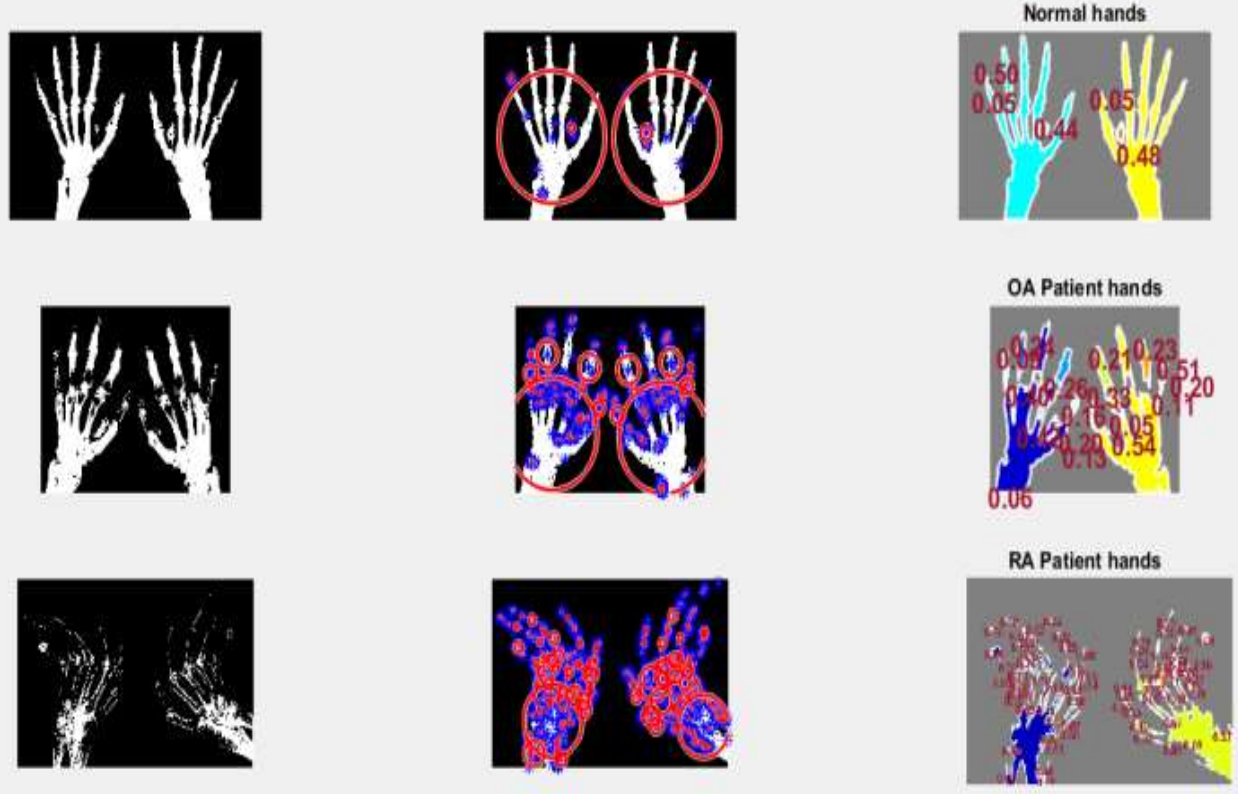

Figure 10. Comparative Image Plot for Normal, OA and RA Patient Hands

The neck is generally having a curvature which is distorted by the disease condition. The neck becomes more stiff and erect as evident from OA and RA patient condition. The 
larger connectivity of area shows the reduced spacing in the vertebral column (neck region) and variation in centroids is different in OA and RA cases.

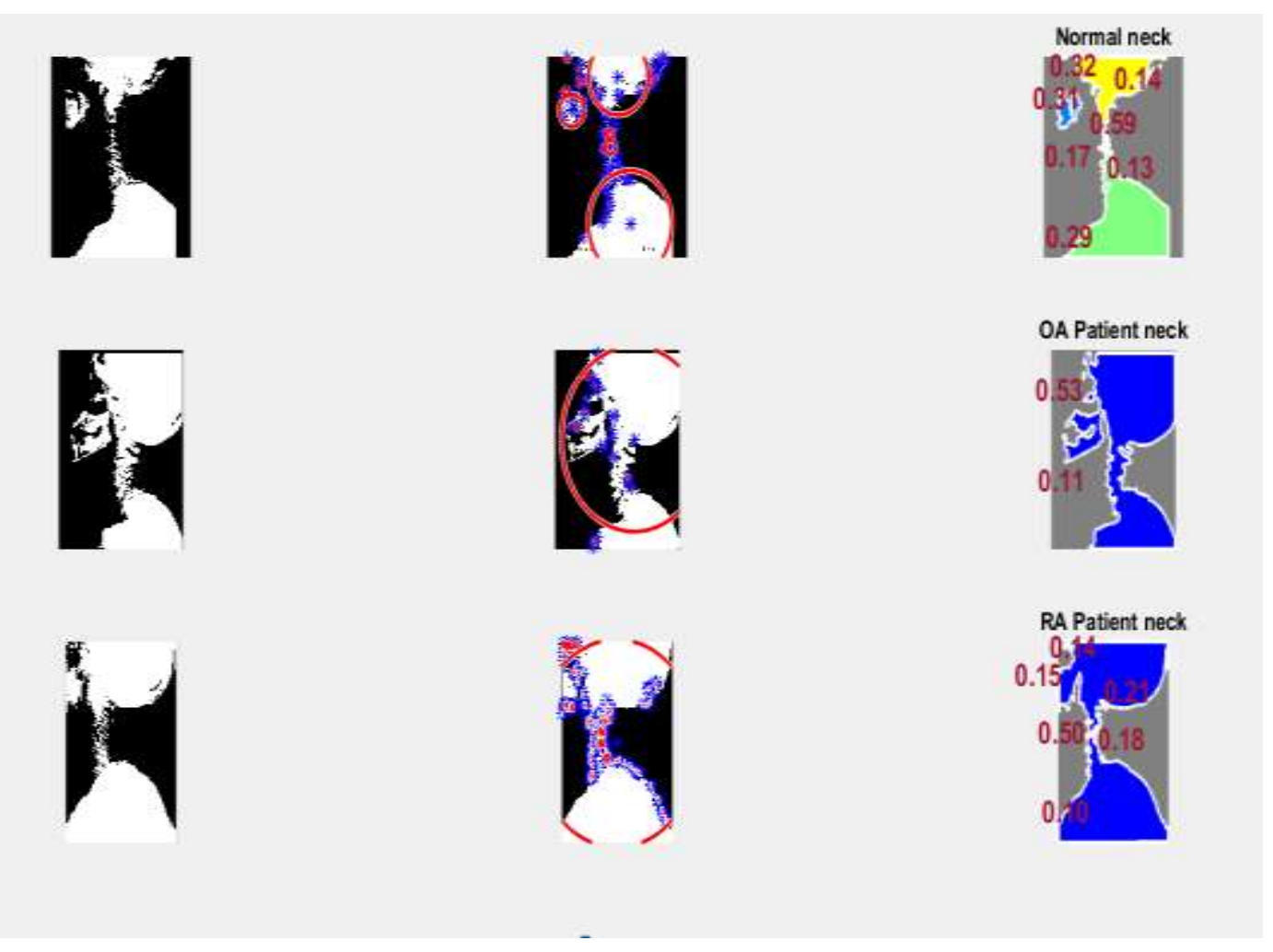

Figure 11. Comparative Image Plot for Normal, OA and RA Patient Neck

\section{Discussion and Conclusion}

Arthritis is a bone related disabling disorder. The field is very diverse and it is hard to classify the kind of arthritis by means of imaging alone. The image processing techniques are thus used to better contemplate the differences and device a possible diagnostic tool.

The Osteoarthritis (OA) is degenerative disorder and Rheumatoid Arthritis (RA) is mainly genetic disorder but the damage to the bones is of fine difference between the two conditions.

The region properties such as centroids, orientation, major axis length, minor axis length, extrema and eccentricity are used in the above study to statistically analyze the differences in the knee, hands and neck regions of the normal, OA and RA patients.

The centroid, orientation, area and boundary identification on the images using MATLAB helps with better insight. The symmetrical and asymmetrical wear and tear is one noteworthy point. The orientation is more evident in the case of RA than OA. The centroids are more likely to form in the greater degree of bone spurring. These findings can help understand better areas of concern to help in formulation of a precise classifier. The image processing technique can be used to better understand the variation amongst the different type's thus enabling stronger grip on the possible pre-requisites for an Arthritis classifier tool. The SVM train-test can be used to program the classifier to recognize the similarities amongst the same kind of arthritis and differentiate from the other kind. This would serve as a differential diagnostic tool to aid the identification of specific type of arthritis from the $\mathrm{x}$-ray image processing. 


\section{References}

[1] M. J. Steinbeck, L. J. Nesti, P. F. Sharkey and J. Parvizi, "Myeloperoxidase and chlorinated peptides in osteoarthritis: potential biomarkers of the disease", Journal of orthopaedic research, vol. 25, no. 9, (2007), pp. 1128-1135.

[2] D. Symmons, G. Turner, R. Webb, P. Asten, E. Barrett, M. Lunt and A. Silman, "The prevalence of rheumatoid arthritis in the United Kingdom: new estimates for a new century", Rheumatology, vol. 41, no. 7, (2002), pp. 793-800.

[3] "Hip dysplasia: a case-control study in adults with severe osteoarthritis", International orthopaedics, vol. 39, no. 4, pp. 793-798.

[4] V. Majithia and S. A. Geraci, "Rheumatoid arthritis: diagnosis and management", The American journal of medicine, vol. 120, no. 11, (2007), pp. 936-939.

[5] A. Mittal and S. K. Dubey, "Analysis of rheumatoid arthritis through image processing", International Journal of Computer Science Issues (IJCSI), vol. 9, no. 6, (2012).

[6] P. D. Tantua, "Intelligent Knee Rheumatoid Arthritis Identification System Based On Image Processing and Neural Classification (Doctoral dissertation", Near East University), (2015).

[7] S. A. Bhisikar and S. N. Kale, "Automatic analysis of rheumatoid Arthritis based on statistical features", In Automatic Control and Dynamic Optimization Techniques (ICACDOT), International Conference on, IEEE, (2016), pp. 242-245.

[8] M. Gobikrishnan, T. Rajalakshmi and U. Snekhalatha, "Diagnosis of rheumatoid arthritis in knee using fuzzy C means segmentation technique", In Communication and Signal Processing (ICCSP), 2016 International Conference on, IEEE, (2016), pp. 0430-0433.

[9] J. Kälvesten, "Automatic image analysis for decision support in rheumatoid arthritis and osteoporosis", Diss. Linköping University Electronic Press, (2015).

[10] Y. Huo, K.L. Vincken, D. van der Heijde, M. J.H. De Hair, F. P. Lafeberand Max A. Viergever, "Automatic quantification of radiographic finger joint space width of patients with early rheumatoid arthriti”, IEEE Transactions on Biomedical Engineering,vol. 63, no. 10, (2016), pp. 2177-2186.

[11] Y. Huo, K. L. Vincken, M. A. Viergever and F. P. Lafeber, "Automatic joint detection in rheumatoid arthritis hand radiographs", In Biomedical Imaging (ISBI), 2013 IEEE 10th International Symposium on, IEEE, (2013), pp. 125-128.

[12] M. Subramoniam and V. Rajini, "Statistical feature based classification of arthritis in knee X-ray images using local binary pattern", In Circuits, Power and Computing Technologies (ICCPCT), 2013 International Conference on, IEEE, (2013), pp. 873-875.

[13] T.-F. Lee, W.-C. Lin, L.-F. Wu and H.-Y. Wang, "Analysis of vibroarthrographic signals for knee osteoarthritis diagnosis", In Genetic and Evolutionary Computing (ICGEC), 2012 Sixth International Conference on, IEEE, (2012), pp. 223-228.

\section{Authors}

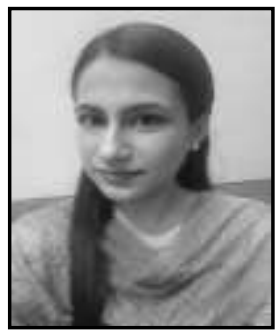

Hunza Hayat, she is currently Masters Student at Department of Biomedical Engineering and Sciences within NUST School of Mechanical and Manufacturing Engineering (SMME). Her research interests include biomedical imaging.

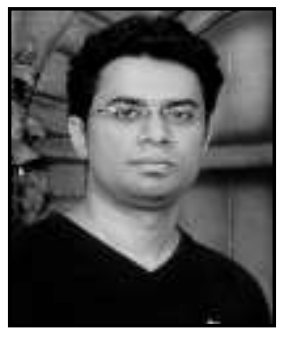

Syed Omer Gilani, he is currently an assistant professor at National University of Sciences and Technology (NUST), Pakistan. He earned his Ph.D. in electrical and computer engineering from National University of Singapore in 2013 and MSc degree in computer engineering from Sweden in 2006. Between 2006 and 2008, he worked at Interactive Multimedia Lab (Singapore). His research interests include human-machine interaction and networking and actively consults for industry on various projects. 


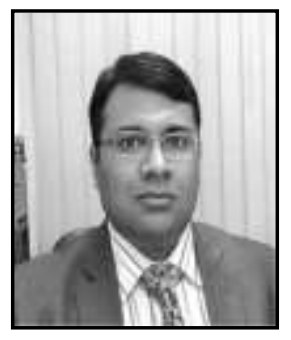

Mohsin Jamil, he is currently assistant professor and Dy Head of Department at National University of Sciences and Technology (NUST), Islamabad, Pakistan. He received his BEng Industrial Electronics degree from NED University, Pakistan, in 2004. He is MSc Electrical Engineering degree holder from Dalarna University Sweden and National University of Singapore in controls and automation. He received $\mathrm{PhD}$ Degree from University of Southampton, UK. His research interests include digital control design and smart grid technologies. He is author of a book chapter and several IEEE publications. 